\title{
Locally compact products and coproducts in categories of topological groups
}

\section{Karl Heinrich Hofmann and Sidney A. Morris}

\begin{abstract}
In the category of locally compact groups not all families of groups have a product. Precisely which families do have a product and a description of the product is a corollary of the main theorem proved here. In the category of locally compact abelian groups a family $\left\{G_{j} ; j \in J\right\}$ has a product if and only if all but a finite number of the $G_{j}$ are of the form $K_{j} \times D_{j}$, where $K_{j}$ is a compact group and $D_{j}$ is a discrete torsion free group. Dualizing identifies the families having coproducts in the category of locally compact abelian groups and so answers a question of $Z$. Semaden $i$.
\end{abstract}

It is well known that if $C$ is the category of all topological groups (hereinafter referred to as TopGps), of all abelian topological groups, of all compact groups, or of all compact abelian groups with the morphisms being the continuous homomorphisms then, for any family $\left\{G_{j} ; j \in J\right\}$ of members of $C$, both the product $\prod_{j \in J}^{C} G_{j}$ and the coproduct $\frac{1}{j \in J}_{j}^{C} G_{j}$ exist. However Semadeni [2, 3] notes that very little is known about coproducts (he could also have said products) in the category LCA of

Received 29 June 1977. The first author's research was done while he was a Visiting Professor at La Trobe University and a guest of the Australian Mathematical Society. 
locally compact abelian groups. It is clear that if $\left\{G_{j} ; j \in J\right\}$ is a family in LCA such that all but a finite number of $G_{i}$ are discrete, then $\frac{L C A}{j \in J} G_{j}$ exists. Semadeni [3, Problem 490] asks if there is an infinite family $\left\{G_{j} ; j \in J\right\}$ of non-discrete members of $L C A$ such that $\int_{j \in J}^{L C A} G_{j}$ exists. We give an affirmative answer and characterize those families $\left\{G_{j} ; j \in J\right\}$ for which $\frac{L C A}{j \in J} G_{j}$ exists. This is done by solving the dual problem of describing products in LCA which is a corollary of our main result.

PROPOSITION 1. If $\mathrm{C}$ is a full subcategory of TOpGps which has finite limits, then the finite products in $C$ are the finite products in TopGps.

Proof. It suffices to prove that if $A$ and $B$ are in $C$, then their product in $C, A \times{ }_{C} B$, is topologically isomorphic to $A \times B$, their product in TopGps.

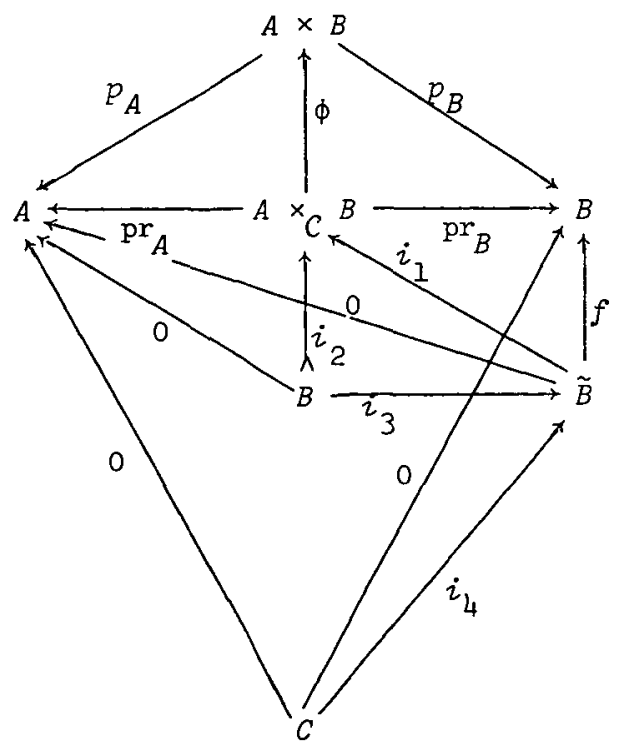

Let ${ }^{\mathrm{pr}}{ }_{A}$ and $\mathrm{pr}_{B}$ be the projections of $A \times_{C} B$ into $A$ and $B$, 
respectively. Also let $p_{A}$ and $p_{B}$ be the projections of $A \times B$ into $A$ and $B$ respectively. As $A \times B$ is the product in TopGps, there exists a continuous homomorphism $\phi: A \times{ }_{C} B \rightarrow A \times B$ such that $\operatorname{pr}_{A}=p_{A} \phi$ and $\mathrm{pr}_{B}=p_{B} \phi$. Let $\tilde{B}$ be the kernel of $\mathrm{pr}_{A}$, and $i_{I}$ the inclusion of $\tilde{B}$ in $A \times_{C} B$. Let $f: \tilde{B} \rightarrow B$ be $\operatorname{pr}_{B} i_{1}$.

The identity map $1: B \rightarrow B$ and the trivial map $0: B \rightarrow A$ imply the existence of a continuous homomorphism $i_{2}$ of $B$ into $A{ }{ }_{C} B$ which is a topological embedding. As $\operatorname{pr}_{A} i_{2}=0$, the definition of $\tilde{B}$ implies the existence of a continuous homomorphism $i_{3}: B \rightarrow \tilde{B}$ which is a topological embedding.

Let $C$ be the kernel of $f$. So $i_{4}$ is the inclusion map of $C$ in $\tilde{B}$ and $f i_{4}$ is the trivial map of $C$ into $B$. Mapping $C$ into $\tilde{B}$ by $i_{4}$ and $\tilde{B}$ into $A$ by 0 yields the trivial map $0: C \rightarrow A$. The pair of maps $0: C \rightarrow A$ and $0: C \rightarrow B$ yield a unique continuous homomorphism $\theta: C \rightarrow A \times_{C} B$ such that $\operatorname{pr}_{A} \Theta=0$ and $\operatorname{pr}_{B} \Theta=0$. But there are two maps with this property, $0: C \rightarrow A \times_{C} B$ and $i_{1} i_{4}: C \rightarrow A \times_{C} B$. So $i_{4}=0$ and thus $C$ is the trivial group. Hence $f$ is injective. But as $f i_{3}=1, f$ is also a retraction and hence $f$ is a topological group isomorphism and $\tilde{B}$ is topologically isomorphic to $B$.

Similarly, if we put $\tilde{A}$ equal to the kernel of $\mathrm{pr}_{B}$, then we can show $\left.\operatorname{pr}_{A}\right|_{\tilde{A}}$ is a topological isomorphism of $\tilde{A}$ onto $A$.

We show next that $A \times{ }_{C} B=\tilde{A} \tilde{B}$ and $\tilde{A} \cap \tilde{B}=\{1\}$; 


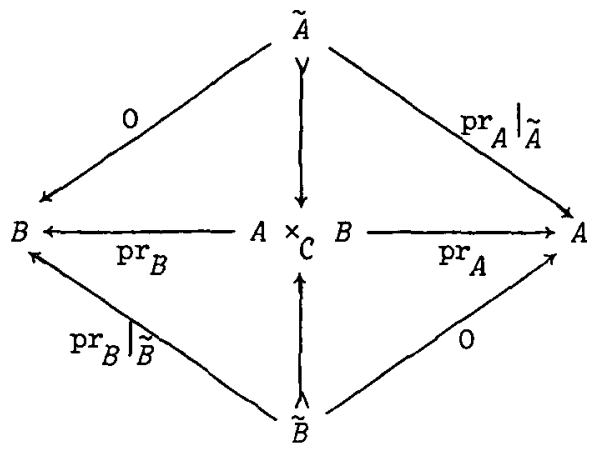

Let $g \in \tilde{A} \cap \tilde{B}$. As $g \in \tilde{B}, \operatorname{pr}_{A}(g)=1$. But $\left.\operatorname{pr}_{A}\right|_{\tilde{A}}$ is an isomorphism and so $g=1$. So $\tilde{A} \cap \tilde{B}=\{1\}$.

Now if $x \in A \times{ }_{C} B$, then $h=\left(\operatorname{pr}_{A} \mid \tilde{A}\right)^{-1} \operatorname{pr}_{A}(x) \in \tilde{A}$. So $\operatorname{pr}_{A}(h)=\operatorname{pr}_{A}(x)$. Thus $\operatorname{pr}_{A}\left(h^{-1} x\right)=1$, which implies $h^{-1} x \in \operatorname{ker}\left(\operatorname{pr}_{A}\right)=\tilde{B}$ Hence $x \in \tilde{A} \tilde{B}$ and $A \times_{C} B=\tilde{A} \tilde{B}$.

As $\tilde{A}$ and $\tilde{B}$ are normal subgroups of $A \times{ }_{C} B$ and $A \times_{C} B=\tilde{A} \tilde{B}$ and $\tilde{A} \cap \tilde{B}=\{1\}$, we have that $A \times{ }_{C} B$ is algebraically isomorphic to $A \times B$. More precisely $\phi$ is an algebraic isomorphism of $A \times{ }_{C} B$ onto $A \times B$.

Now define $\psi: A \times B+A \times{ }_{C} B$ by

$$
\psi(a, b)=\left[\left(\operatorname{pr}_{A} \mid \tilde{A}\right)^{-1} a\right]\left[\left(\left.\operatorname{pr}_{B}\right|_{\tilde{B}}\right)^{-1} b\right] \text {. }
$$

If $g \in A \times{ }_{C} B$, then $g \in \tilde{A} \tilde{B}$; that is, $g=\tilde{a} \tilde{b}$ with $\tilde{a} \in \tilde{A}$ and $\tilde{b} \in \tilde{B}$. Then $\phi(g)=\left(\operatorname{pr}_{A}(\tilde{a}), \operatorname{pr}_{B}(\tilde{b})\right)$. Clearly $\phi$ and $\psi$ are inverse maps. As $\psi$ is also continuous, $\phi$ is a topological group isomorphism and $A \times{ }_{C} B$ is topologically isomorphic to $A \times B$.

REMARKS. It is not necessary to assume $\mathcal{C}$ is a full subcategory of TopGps in Proposition 1. It suffices to assume that $C$ has identity arrows, 0 arrows, and inclusion arrows.

The reader should be cautioned not to assume a priori that, in proofs like the preceding and in the discussions which follow, the products we consider are defined on set theoretical cartesian products. 
LEMMA 1. Let $\mathrm{C}$ be a full subcategory of TopGps which has finite limits. If $F$ is a finite subset of a set $J$ and $\prod_{j \in J}^{C} G_{j}$ exists, then $\prod_{j \in J \backslash F}^{C} G_{j}$ exists and

$$
\prod_{j \in J}^{C} G_{j} \simeq \prod_{j \in F}^{C} G_{j} \times \prod_{j \in \mathcal{J} \backslash F}^{C} G_{j}
$$

Proof. Let $K$ be the kernel of the canonical map $\theta$ of $\prod_{j \in J}^{C} G_{j}$ into $\prod_{j \in F}^{C} G_{j}$. We claim that $K=\prod_{j \in J \backslash F}^{C} G_{j}$, with the projections of $K$ into $G_{j}$ being the projections of $\prod_{j \in J}^{C} G_{j}$ (for $j \in J \backslash F$ ) restricted to $K$.

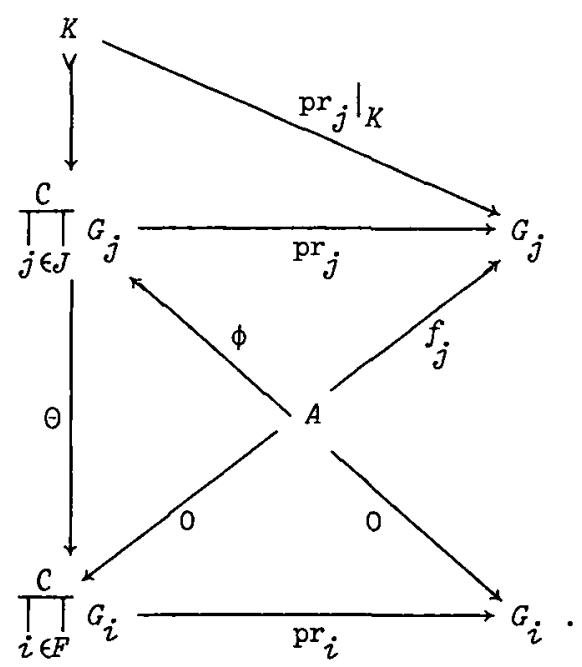

Let $A$ be any member of $C$ and $f_{j}, j \in J \backslash F$, a family of continuous homomorphisms of $A$ into $G_{j}$. Define $f_{i}: A \rightarrow G_{i}, i \in F$, to be the trivial homomorphisms. Then there exists a continuous homomorphism $\phi$ of $A$ into $\prod_{j \in J}^{C} G_{j}$ such that $\operatorname{pr}_{j} \phi=f_{j}, j \in J$. As 
$\operatorname{pr}_{i}^{\phi}=0, \quad i \in F, \quad \theta \phi=0$, and so $\phi(A) \subseteq K$. Hence $K=\prod_{j \in J \backslash F}^{C} G_{j}$. Once we know $\prod_{j \in \mathcal{J} \backslash F}^{C} G_{j}$ exists, the usual categorical argument shows that $\prod_{j \in J}^{C} G_{j}$ is topologically isomorphic to $\prod_{j \in F}^{C} G_{j} \times{ }_{C} \underset{j \in J \backslash F}{C} G_{j}$, which by Proposition 1 , is the required resi.:

PROPOSITION 2. Let $\because$ be a full subcategory of TopGps which is complete and whose objects are compact. If every compact subgroup of a topological group in $K$ is also in $K$, tinen for any family $\left\{G_{j} ; j \in J\right\}$ in $K, \prod_{j \in J}^{K} G_{j} \simeq \prod_{j \in J} G_{j}$; that $i s$, the product in $K$ and the product in TopGps coincide.

Proof.

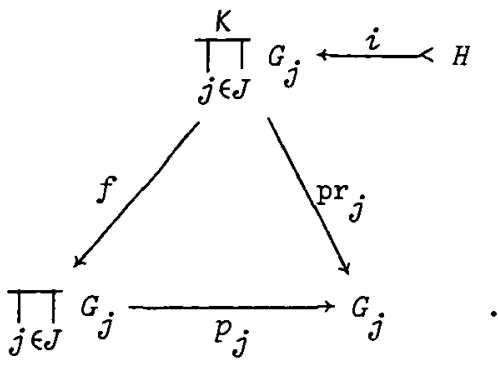

Let $p_{j}$ and $\operatorname{pr}_{j}$ be the projections or $\prod_{j \in J} G_{j}$ into $G_{j}$ and $\prod_{j \in J}^{K} G_{j}$ into $G_{j}$, respectively. As $\prod_{j \in J} G_{j}$ is the product in TopGps of $\left\{G_{j} ; j \in J\right\}$, the maps $\operatorname{pr}_{j}: 7 \prod_{j \in J}^{K} G_{j}+G_{j}$ induce a continuous homomorphism $f: \prod_{j \in J}^{K} G_{j}$ into $\prod_{j \in J} G_{j}$ such that $p_{j} f=\mathrm{pr}_{j}$, for each $j \in J$. Let $x \in \prod_{j \in J}^{K} G_{j}$ be such that $f(x)=1$. Then $p_{j} f(x)=1$ and so $\operatorname{pr}_{j}(x)=1$, for each $j \in J$. Let $H$ be the closed subgroup of $\prod_{j \in J}^{K} G_{j}$, 
generated by $\{x\}$. Then $\operatorname{pr}_{j}(H)=\{1\}$, for all $j \in J$. If $i: H \rightarrow \prod_{j \in J}^{K} G_{j}$ is the inclusion map, then $\operatorname{pr}_{j} i=0=\operatorname{pr}_{j} 0^{\prime}$, where 0 is the trivial map of $H$ into $G_{j}$ and $O^{\prime}$ is the trivial map of $H$ into $\prod_{j \in J}^{K} G_{j}$. As $\prod_{j \in J}^{K} G_{j}$ is the product in $K$, the uniqueness in the product property implies that $i=0^{\prime}$. Thus $B=\{1\}$ and $x=1$. Hence the map $f$ is injective.

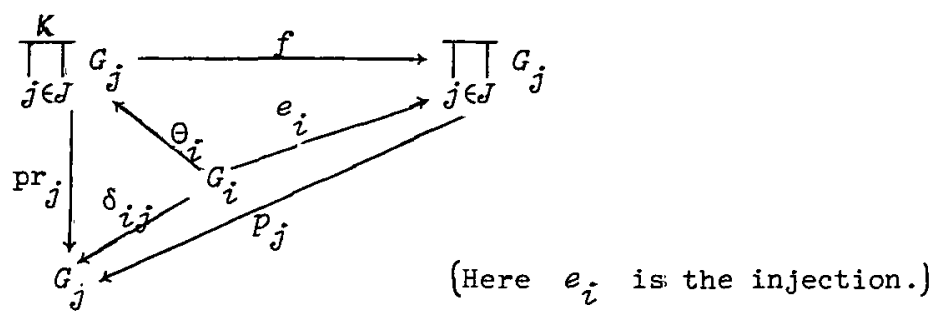

Now let $\delta_{i j}: G_{i} \rightarrow G_{j}$ be the identity map if $i=j$ and the trivial homomorphism if $i \neq j$. Then there exist continuous homomorphisms $\Theta_{i}: G_{i} \rightarrow \prod_{j \in J}^{K} G_{j}$ such that $\delta_{i j}=\operatorname{pr}_{j} \Theta_{i}$ and $\delta_{i j}=p_{j} e_{i}$. $j \in J$. By uniqueness in the product property $f \theta_{i}=e_{i}$ So $f\left(\prod_{j \in J}^{K} G_{j}\right)$ is a compact subgroup of $\prod_{j \in J} G_{j}$ which contains $e\left(G_{i}\right)$ for each $i \in J$. Thus $f\left(\prod_{j \in J}^{K} G_{j}\right)=\prod_{j \in J} G_{j}$ and $f$ is surjective. Hence $f$ is a topological group isomorphism of $\prod_{j \in J}^{K} G_{j}$ onto $\prod_{j \in J} G_{j}$.

LEMMA 2. Let $c$ be a full subcategory of TopGps and $K$ a fuzl subcategory of $\mathrm{C}$ which is complete and whose objects are all compact subgroups of topological groups in $C$. If $K_{j} \in K$, for $j \in J$, then $\prod_{j \in J}^{C} K_{j}$ exists and is topologically isomorphic to $\prod_{j \in J}^{K} K_{j}$. 
Proof. This follows immediately from the fact that $\prod_{j \in J}^{K} K_{j}$ is $\prod_{j \in J} K_{j}$, the product in TopGps of $\left\{K_{j}: j \in \sigma^{\tau}\right\}$.

LEMMA 3. Under the hypotheses of Lemma 2, if $K_{j} \leq G_{j}$, where $K_{j} \in K$ and $G_{j} \in \mathcal{C}$ for $j \in J$ and $\prod_{j \in J}^{C} G_{j}$ exists, then the canonical homomorphism of $\prod_{j \in J}^{\mathcal{C}} K_{j}$ into $\prod_{j \in J}^{C} G_{j}$ is a topological embedding.

Proof. This follows immediately from the fact that $\prod_{j \in J}^{C} K_{j}$ is $\prod_{j \in J} K_{j}$, and so is compact.

LEMMA 4. Let $C$ be a full subcategory of TopGps with finite limits and $K$ a full subcategory of compact $C$-objects which is complete and contains all compact subgroups of groups in C. If $G_{j} \in \mathcal{C}, j \in J$, and $\prod_{j \in J}^{\mathcal{C}} G_{j}$ exists and $K$ is a compact subgroup of $\prod_{j \in J}^{\mathcal{C}} G_{j}$, then $K \leq \prod_{j \in J}^{C} \operatorname{pr}_{j}(K) \leq \prod_{j \in J}^{C} G_{j}$, where $\operatorname{pr}_{j}$ is the cononical projection of $\prod_{j \in J}^{C} G_{j}$ into $G_{j}, j \in J$.

Proof.

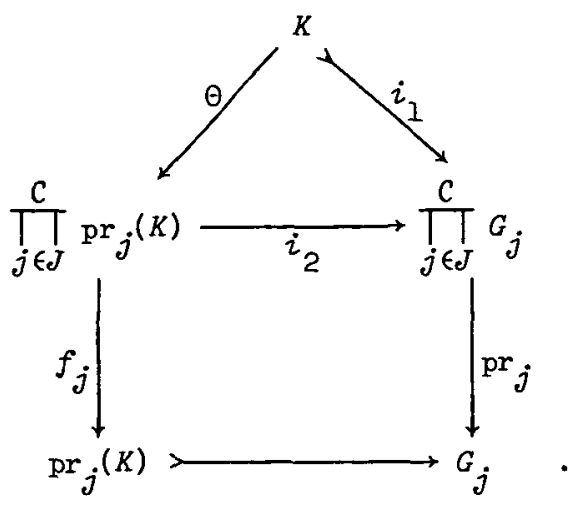


As $K$ is compact, $\operatorname{pr}_{j}(K)$ is compact and so is in $K$. Hence $\prod_{j \in J}^{K} \operatorname{pr}_{j}(K)$ exists and by Lemma 2 is isomorphic to $\prod_{j \in J}^{C} \operatorname{pr}_{j}(K)$. By Lemma 3 , the canonical homomorphism $\prod_{j \in J}^{C} \operatorname{pr}_{j}(K)+\prod_{j \in J}^{C} G_{j}$ is a topological embedding $i_{2}$. The maps $\operatorname{pr}_{j}: K \rightarrow \operatorname{pr}_{j}(K)$ imply the existence of a continuous homomorphism $\theta: K \rightarrow \prod_{j \in \mathcal{J}}^{\mathcal{C}} \operatorname{pr}_{j}(K)$ such that $f_{j} \theta=\operatorname{pr}_{j}$, where $f_{j}$ are the projections of $\prod_{j \in J}^{C} \operatorname{pr}_{j}(K)$. Finally observe that $i_{2} \theta=i_{1}$, the inclusion of $K$ in $\prod_{j \in J}^{C} G_{j}$. So $\theta$ is a topological embedding, as required.

LEMMA 5. If $H_{j}$ and $G_{j}, j \in J$, are topological groups with $H_{j} \leq G_{j}$ and $\prod_{j \in J} H_{j}$ an open subgroup of $\prod_{j \in J} G_{j}$, then there is a finite subset $F$ of $J$ such that for all $j \in J \backslash F, H_{j}=G_{j}$.

Proof. This follows from the definition of the Tychonoff product topology.

LEMMA 6. Let $\mathcal{C}$ be a full subcategory of TopGps which has finite limits. If $G_{j} \in \mathcal{C}, j \in J$, and $\prod_{j \in J}^{C} G_{j}$ exists and is locally compact, then each $G_{j}$ is locally compact and there exists a finite subset $F$ of

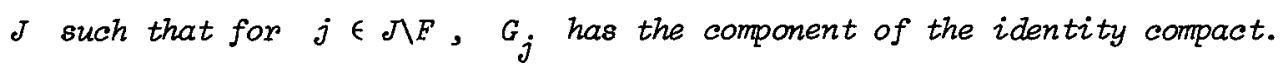

Proof. By Proposition I and Lemma 1 , each $G_{j}$ is locally compact. If none of $G_{j_{1}}, \ldots, G_{j_{n}}$ has compact component then each contains a copy of $R$, the additive group of real numbers with the usual topology. Proposition $I$ and Lemma 1 then imply that $G_{j_{1}} \times G_{j_{2}} \times \ldots \times G_{j_{n}} \leq \prod_{j \in J}^{C} G_{j}$. 
So $\prod_{j \in J}^{C} G_{j}$ has a subgroup topologically isomorphic to $R^{n}$. But no locally compact group can contain $R^{n}$, for arbitrary large $n$. So all but a finite number of the $G_{j}$ have compact components.

LEMMA 7. Let $C$ be a full subcategory of TopGps with finite limits and $K$ a full subcategory of compact $c$-objects which is complete and contains all compact subgroups of groups in $\mathrm{C}$, and let $G_{j} \in \mathcal{C}$, $j \in J$ be such that each $G_{j}$ has $i$ ts connected component $\left(G_{j}\right)_{0}$ compact. If $\prod_{j \in J}^{C} G_{j}$ exists then its component $\left(\prod_{j \in J}^{K}\left(G_{j}\right)\right\}_{0}$ is topologically isomorphic to $\prod_{j \in J}^{K}\left(G_{j}\right)_{0}$.

Proof. By Lemma 3, $\prod_{j \in J}^{K}\left(G_{j}\right)_{0} \leq \prod_{j \in J}^{C} G_{j}$, and as $\prod_{j \in J}^{K}\left(G_{j}\right)_{0}$ is connected, $\prod_{j \in J}^{\therefore}\left(G_{j}\right)_{0} \leq\left(\prod_{j \in J}^{\mathcal{C}} G_{j}\right)_{0}$.

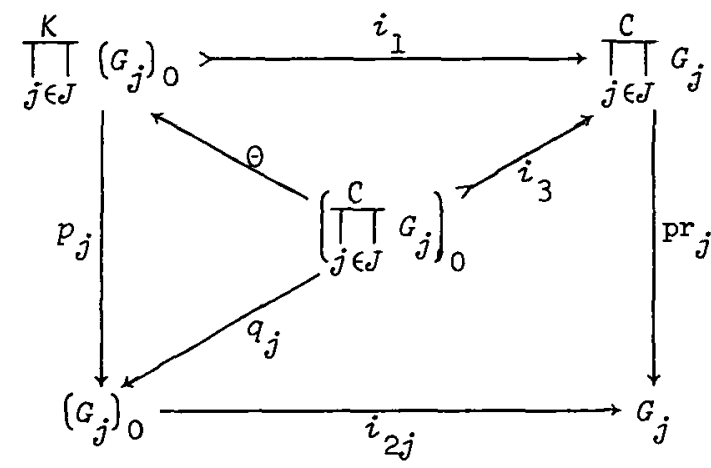

Let $i_{1}$ be the inclusion map of $\prod_{j \in J}^{K}\left(G_{j}\right)_{0}$ in $\prod_{j \in J}^{C} G_{j}, i_{2 j}$ the inclusion map of $\left(G_{j}\right)_{0}$ in $G_{j}, \mathrm{pr}_{j}$ the projection of $\prod_{j \in J}^{C} G_{j}$ into $G_{j}, i_{3}$ the inclusion map of $\left(\prod_{j \in J}^{C} G_{j}\right)_{0}$ in $\prod_{j \in J}^{C} G_{j}, q_{j}$ the 
restriction of $\operatorname{pr}_{j}$ to $\left(\prod_{j \in J}^{C} G_{j}\right)_{0}$ and $p_{j}$ the projection of $\prod_{j \in J}^{K}\left(G_{j}\right)_{0}$ into $\left(G_{j}\right)_{0}$. As $\left\{\prod_{j \in J}^{C} G_{j}\right\}_{0}$ is connected and $q_{j}$ is continuous, $q_{j}\left(\left(\prod_{j \in J}^{C} G_{j}\right)_{0}\right) \leq\left(G_{j}\right)_{0}$. The family of maps $q_{j}$ determine a continuous homomorphism $\theta:\left(\prod_{j \in J}^{\mathcal{C}} G_{j}\right)_{0} \rightarrow \prod_{j \in J}^{K}\left(G_{j}\right)_{0}$ such that $p_{j} \theta=q_{j}$ for each $j \in J$. The family of maps $i_{2 j} q_{j}$ of $\left(\prod_{j \in J}^{C} G_{j}\right)_{0}$ into $G_{j}, j \in J$, determine a unique continuous homomorphism $\delta:\left(\prod_{j \in J}^{\mathcal{C}} G_{j}\right)_{0} \rightarrow \prod_{j \in J}^{\mathcal{C}} G_{j}$ such that $\operatorname{pr}_{j} \delta=i_{2 j} q_{j}$. But the maps $i_{3}$ and $i_{1} \theta$ both have this property and thus $i_{3}=i_{1} \theta$. Hence $\left(\prod_{j \in J}^{\mathcal{C}} G_{j}\right)_{0}=\prod_{j \in J}^{K}\left(G_{j}\right)_{0}$.

LEMMA 8. Under the hypotheses of Lemma 7, if $\prod_{j \in J}^{\mathcal{C}} G_{j}$ exists and is locally compact, then $\prod_{j \in J}^{\mathcal{C}} G_{j}$ has a compact open subgroup $\prod_{j \in J}^{K} H_{j}$; where each $H_{j}$ is an open subgroup of $G_{j}$.

Proof. Lemma 7 says that $\prod_{j \in J}^{\mathcal{C}} G_{j}$ is a locally compact group with compact component and so $\prod_{j \in J}^{C} G_{j}$ has a compact open subgroup $C$. Putting $H_{j}=\operatorname{pr}_{j}(C)$, where $\operatorname{pr}_{j}$ is the canonical map of $\prod_{j \in d}^{C} G_{j}$ into $G_{j}$, we have that $H_{j}$ is in $K$ and by Lemma $4, \quad c \leq \prod_{j \in J}^{K} H_{j} \leq \prod_{j \in J}^{C} G_{j}$. So $\prod_{j \in J}^{K} H_{j}$ is a compact open subgroup of $\prod_{j \in J}^{\mathcal{C}} G_{j}$. By Proposition $I$ and Lemma $I, \quad \mathrm{Pr}_{j}$ 
is a retraction and thus a quotient mapping. So each $H_{j}$ is an open subgroup of $G_{j}$.

LEMMA 9. Under the hypotheses of Lemma 7 if $\prod_{j \in J}^{C} G_{j}$ exists and is Locally compact, there exists a finite subset $F$ of $J$ such that for $j \in \lambda F$, the group $G_{j}$ has a compact open normal subgroup $H_{j}$ with $H_{j}$ a maximal compact subgroup of $G_{j}$.

Proof. Let $H_{j}$ be as in Iemma 8 . Let $F_{1}$ be the subset of $J$ consisting of those $j$ for which there is a compact group $C_{j}$ with $H_{j} \lessgtr C_{j} \leq G_{j}$. For $j \in J \backslash F_{I}$, set $C_{j}=H_{j}$. Then $\prod_{j \in J}^{K} H_{j} \leqq \prod_{j \in J}^{K} c_{j} \leqq \prod_{j \in J}^{C} G_{j}$. So $\prod_{j \in J}^{K} H_{j}$ is an open subgroup of $\prod_{j \in J}^{K} c_{j}$. By Lemma $5, F_{1}$ is finite.

Now set $F_{2}=\left\{j \in J: H_{j}\right.$ is not normal in $\left.G_{j}\right\}$. So for each $j \in F_{2}$, there exists a $g_{j} \in G_{j}$ such that $H_{j} \neq g_{j}{ }_{j} g_{j}^{-1}$. For $j \in J \backslash F_{2}$, set $g_{j}=1$. Define an automorphism $\alpha$ of $\prod_{j \in J}^{C} G_{j}$ by the diagram

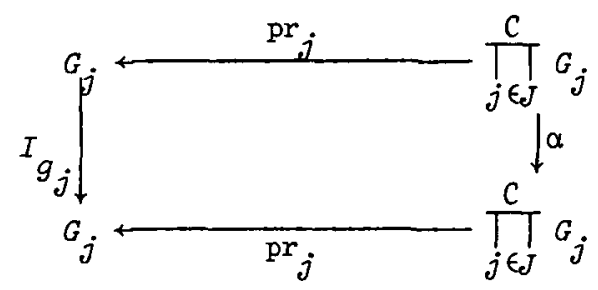

where $I_{g_{j}}(x)=g_{j} x g_{j}^{-1}$ for $x \in G_{j}$, and $\mathrm{pr}_{j}$ is the projection. Then $\alpha\left(\prod_{j \in J}^{K} H_{j}\right\}$ is open in $\prod_{j \in J}^{C} G_{j}$. But

$$
\operatorname{pr}_{j} \alpha\left(\prod_{j \in J}^{K} H_{j}\right)=I_{g_{j}} \operatorname{pr}_{j}\left(\prod_{j \in J}^{K} H_{j}\right)=g_{j} h_{j} g_{j}^{-1} \text {. }
$$


So

$$
\alpha\left(\prod_{j \in J}^{K} H_{j}\right) \leq \prod_{j \in J}^{K}\left(g_{j} H_{j} g_{j}^{-1}\right) \leq \prod_{j \in J}^{C} G_{j}
$$

and thus $\prod_{j \in J}^{K}\left(g_{j} H_{j} g_{j}^{-I}\right)$ is open in $\prod_{j \in J}^{C} G_{j}$. Hence

$$
\left(\prod_{j \in J}^{K} H_{j}\right) \cap\left(\prod_{j \in J}^{K} g_{j}{ }_{j} g_{j}^{-I}\right)=\prod_{j \in J}^{K}\left(H_{j} \cap g_{j}{ }_{j} g_{j}^{-1}\right)
$$

is open in both $\prod_{j \in J}^{K} H_{j}$ and $\prod_{j \in J}^{K} g_{j}{ }_{j} g_{j}^{-1}$. By Lemma $5, H_{j} \cap g_{j} H_{j} g_{j}^{-1}=H_{j}$ for all but a finite number of $j$ and also $H_{j} \cap g_{j}{ }_{j} g_{j}^{-1}=g_{j} H_{j} g_{j}^{-1}$ for all but a finite number of $j$. Hence $F_{2}$ is finite. The required set $F$ is $F_{1} \cup F_{2}$.

NOTATION. Let $K_{j}$ be a compact open normal subgroup of a topological group $G_{j}$, for each $j \in J$. Let $\prod_{j \in J} K_{j}$ be the product of the $K_{j}$ in TopGps. We denote by $\prod_{j \in J}\left\langle G_{j} ; K_{j}\right\rangle$ the topological group which has as its underlying group the cartesian product $\prod_{j \in J} G_{j}$ and whose topology is determined by letting $\prod_{j \in J} K_{j}$, with its Tychonoff product topology, be an open subgroup. So $\prod_{j \in J}\left(G_{j} ; K_{j}\right)$ is a locally compact group.

LEMMA 10 . Under the hypotheses of Lemma 7 , if each $K_{j}$ is a compact open normal subgroup of $G_{j}$ such that $G_{j} / K_{j}$ is torsion free and $\prod_{j \in J}\left\langle G_{j} ; K_{j}\right\rangle$ is in $C$, then $\prod_{j \in J}\left\langle G_{j} ; K_{j}\right\rangle$ is the product of $\left\{G_{j} ; j \in J\right\}$ in $L$, the full subcategory of $C$ whose objects are the locally compact C-objects. 
Proof.

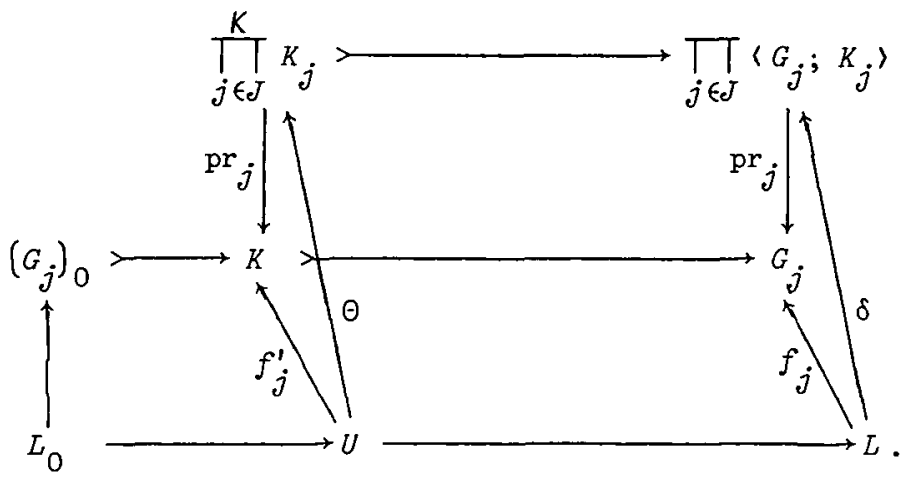

Let $L$ be any locally compact group in $C$ and $f_{j}$ a family of continuous homomorphisms of $L$ into $G_{j}$. Let $L_{0}$ be the component of $I$ and $\left(G_{j}\right)_{0}$ the component of $G_{j}$. As $K_{j}$ is open in $G_{j},\left(G_{j}\right)_{0} \leq K_{j}$. Clearly $f_{j}\left(L_{0}\right) \leq\left(G_{j}\right)_{0}$. Let $U$ be an open subgroup of $L$ such that $U / L_{0}$ is compact.

Consider the diagram

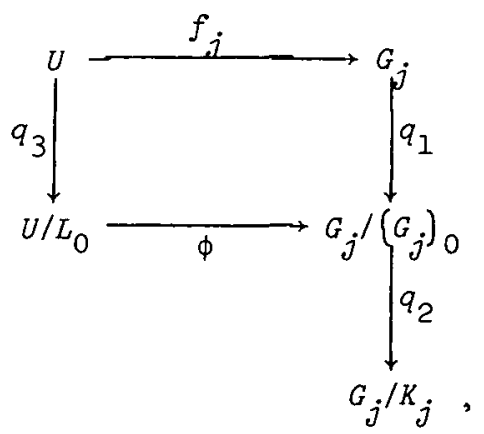

where $q_{1}, q_{2}$, and $q_{3}$ are the natural quotient maps. As $f_{j}\left(L_{0}\right) \leq\left(G_{j}\right)_{0}$, there is an induced continuous homomorphism $\phi: U / L_{0}+G_{j} /\left(G_{j}\right)_{0}$. Since $U / L_{0}$ is compact and $G_{j} / K_{j}$ is torsion free and discrete, $q_{2} \phi\left(U / L_{0}\right)=\{1\}$. Hence $f_{j}(U) \leq K_{j}$.

Let $f_{j}^{\prime}$ be the restriction of $f_{j}$ to $U$. The family of maps 
$f_{j}^{\prime}: U \rightarrow K_{j}$ determine a continuous homomorphism $\theta: U \rightarrow \prod_{j \in J}^{K} K_{j}$ such that $\operatorname{pr}_{j} \Theta=f_{j}^{\prime}$, for each $j \in J$. The maps $f_{j}$ of $L$ into $G_{j}$ determine a (not necessarily continuous) homomorphism $\delta: L+\prod_{j \in J}\left(G_{j} ; K_{j}\right)$ such that $\operatorname{pr}_{j} \delta=f_{j}, j \in J$. Noting that $\left.\delta\right|_{U}=\theta$ and that $\theta$ is continuous on the open subgroup $U$ of $L$, we have that $\delta$ is continuous, and the proof of the lemma is complete.

THEOREM. Let $c$ be a fulz subcategory of TopGps with finite limits and $K$ a full subcategory of $\mathrm{C}$ which is complete and has as its objects all compact subgroups of topological groups in $C$.

(i) Let $\left\{G_{j} ; j \in J\right\}$ be a family in $C$ such that $\prod_{j \in J}^{\mathcal{C}} G_{j}$ exists and is locally compact. Then there exists a finite subset $F$ of $J$ such that for each $j \in \lambda I$, the group $G_{j}$ has a compact open normal subgroup $K_{j}$ with $G_{j} / K_{j}$ torsion free such that

$$
\prod_{j \in J}^{C} G_{j} \simeq \prod_{j \in F} G_{j} \times \prod_{j \in \mathcal{N} \backslash F}\left\langle G_{j} ; K_{j}\right\rangle
$$

where $\prod_{j \in F}$ and $x$ denote the usual product in TopGps.

(ii) Let $L$ be the fulz subcategory of $C$ having as its objects all locally compact groups in $C$. If $\left\{G_{j} ; j \in J\right\}$ is a family of topological groups in $L$ such that for each $j \in \lambda F$, where $F$ is a finite set, $G_{j}$ has a compact open normal subgroup $K_{j}$ with $G_{j} / K_{j}$ torsion free, and if

$\prod_{j \in \mathcal{J}}\left\langle G_{j} ; K_{j}\right\rangle$ is in $L$ then

$$
\prod_{j \in F} G_{j} \times \prod_{j \in J \backslash F}\left(G_{j} ; K_{j}\right\rangle
$$

is the product in $L$ of $\left\{G_{j} ; j \in J\right\}$.

COROLLARY 1. Let $L C$ be the category consisting of all locally compact groups and all continuous homomorphisms between them. Let 
$\left\{G_{j} ; j \in J\right\}$ be a family of topological groups in LC. Then $\prod_{j \in J}^{L C} G_{j}$ exists if and only if $J$ has a finite subset $F$ such that for each $j \in \lambda F, G_{j}$ has a compact open normal subgroup $K_{j}$ such that $G_{j} / K_{j}$ is torsion free.

COROLLARY 2. Let LCA be the category consisting of all locally compact abelian groups and all continuous homomorphisms between them. Let $\left\{G_{j} ; j \in J\right\}$ be a family of topological groups in LCA. Then $\prod_{j \in J}^{L C A} G_{j}$ exists if and only if $J$ has a finite subset $F$, such that for each $j \in \lambda F$, the group $G_{j}$ is topologically isomorphic to $K_{j} \times D_{j}$, where $K_{j}$ is a compact group and $D_{j}$ is a discrete torsion free group. Further if $\prod_{j \in J}^{L C A} G_{j}$ exists, then it is topologically isomorphic to $\prod_{j \in F} G_{j} \times \prod_{j \in \mathcal{}} K_{j} \times \prod_{j \in \mathcal{D}}^{D} D_{j}$, where $\prod$ denotes the usual product in TopGps and $\prod_{j \in \mathcal{\lambda} F}^{D} D_{j}$ denotes the cartesian product group $\prod_{j \in \mathcal{\lambda} \backslash F} D_{j}$ with the discrete topology.

By dualizing Corollary 2 we obtain

COROLLARY 3. Let LCA be the category consisting of all locally compact abelian groups and all continuous homomorphisms between them. Let $\left\{G_{j} ; j \in J\right\}$ be a family of topological groups in LCA. Then the coproduct $\frac{L C A}{j \in J} G_{j}$ exists if and only if $J$ has a finite subset $F$ such that for each $j \in \mathcal{N}$, the group $G_{j}$ is topologically isomorphic to $A_{j} \times B_{j}$, where $A_{j}$ is a discrete group and $B_{j}$ is a compact connected group. Further if $\frac{L C A}{j \in J} G_{j}$ exists, then it is topologically isomorphic to $\prod_{j \in J} G_{j} \times \sum_{j \in \mathcal{D} F}^{D} A_{j} \times \frac{1}{j \in \mathcal{N} F}^{K} B_{j}$ where $\sum_{j \in \mathcal{D} F}^{D} A_{j}$ denotes the restricted 
direct sum of the $A_{j}$ with the discrete topology and $\frac{1}{j \in J \backslash F}_{j}^{K} B_{j}$ the coproduct in the category of compact abelian groups of the $B_{j} ;$ that is, $\stackrel{1}{j \in J \backslash F}_{K}^{K} B_{j}$ is $\left(\prod_{j \in J \backslash F}^{D} \hat{B}_{j}\right)^{\wedge}$, where $\wedge$ denotes the dual group.

LEMMA 11. Let $G$ be a closed subgroup of $K \times D$, where $K$ is a compact abelian group and $D$ is a discrete torsion free abei: i group. Then $G$ is topologically isomorphic to $K_{1} \times D_{1}$, where $K_{1}$ is a compact group and $D_{1}$ is a torsion free group.

Proof. By duality $\hat{G}$ is a quotient group of $\hat{K} \times \hat{D}$, where $\hat{K}$ is a discrete group and $\hat{D}$ is a compact connected group. So $\hat{G}$ is a quotient group of $F \times \hat{D}$, where $F$ is a (suitable) discrete free abelian group. As $\hat{D}$ is divisible, Proposition 5 of [1] implies that $\hat{G}$ is topologically isomorphic to the product of a compact connected group and a discrete group. Dualizing again yields the required result.

COROLLARY 4. The largest full subcategory of LCA which contains the category of all compact abelian groups and has products (and coproducts) and kermels is the category whose objects are all those topological groups of the form $K \times D$, where $K$ is a compact abelian group and $D$ is a discrete torsion free abelian group.

\section{References}

[1] Ronald Brown, Philip J. Higgins and Sidney A. Morris, "Countable products and sums of lines and circles: their closed subgroups, quotients and duality properties", Math. Proc. Cambridge Philos. Soc. 78 (1975), 19-32.

[2] Z. Semadeni, "Projectivity, injectivity and duality", Rozproavy Mat. 35 (1963), 47pp.

[3] Z. Semádeni, Problème P490, Colzoq. Math. 13 (1964-1965), 127.

Department of Mathematics, Tulane University, New Orleans, Louisiana, USA;
Department of Mathematics, La Trobe University, Bundoora, Victoria. 